\title{
Radial Turbine Preliminary Aerodynamic Design Optimization for Expander Cycle Liquid Rocket Engine
}

\author{
Yolanda Mack ${ }^{*}$, Raphael Haftka ${ }^{\dagger}$, Lisa Griffin ${ }^{\ddagger}$, Lauren Snellgrove ${ }^{\S}$, Daniel Dorney ${ }^{* *}$, \\ Frank Huber ${ }^{\dagger \dagger}$ and Wei Shyy",
}

\begin{abstract}
A response surface-based dual-objective design optimization was conducted in the preliminary design of a compact radial turbine for an expander cycle rocket engine. The optimization objective was to increase the efficiency of the turbine while maintaining low turbine weight. Polynomial response surface approximations were used as surrogates, and the accuracies of such approximations improve by limiting the size of the domain and the number of variables for each response of interest. The optimization was accomplished in three stages using an approximate, one-dimensional model. In the first stage, a relatively small number of points were used to identify approximate constraint boundaries of the feasible domain and to reduce the number of variables used to approximate each one of the constraints. In the second stage, a moderate number of points in this approximate feasible domain were used to identify the region where both objectives had reasonable values. The last stage focused on obtaining high accuracy approximation in the region of interest with large number of points. The approximations were used to identify the Pareto front and to perform a global sensitivity analysis. Significant improvement was achieved compared to a baseline design.
\end{abstract}

\section{Introduction}

$\mathrm{I}_{\mathrm{c}}^{\mathrm{N}}$ $\mathrm{N}$ rocket engines, a turbine is used to drive the pumps that deliver fuel and oxygen to the combustion chamber. Cryogenic fuel is heated, resulting in a phase change to a gaseous state. The increased pressure drives the turbine. However, the survivability of turbine blades limits the degree to which the fuel can be heated. In a gas generator cycle, the fuel is preheated using a gas generator. The heated, vaporized gas is sent through the turbine and then to the combustion chamber. In a preburner cycle such as the Space Shuttle Main Engine, both the fuel and oxidizer are preheated using separate preburners to run individual fuel pumps ${ }^{1}$. An expander cycle is a type of rocket engine cycle with improved fuel delivery efficiency as compared to other rocket engine cycles. This is due to that fact that it does not require a portion of the fuel to run the fuel and oxidizer pumps. It has an advantage over the preburner or gas generator cycle because it does not need a second combustion device. Instead, the fuel is heated using waste heat from the thrust chamber cooling jacket. Because the expander cycle has fewer components, engine reliability is increased.

To help reduce the complexity of future engines, NASA is considering using an expander cycle. The expander cycle eliminates the need for a preburner or a gas generator, thus resulting in a simpler and more reliable system. Current expander cycle rocket engines in use are Pratt and Whitney's RL-60 and the Ariane 5 ESC-B. An expander cycle schematic is shown in Figure 1. In the expander cycle, the unburned fuel is preheated by being passed through tubes used to cool the combustion chamber. This heated gas is passed through the turbine used to drive the fuel and oxidizer pumps. The fuel is then sent to the combustion chamber where it is combusted. In the expander cycle, the engine is limited by the amount of power that the turbine can deliver. There is a limited amount of heat available

*Graduate Student Assistant, University of Florida, Gainesville, FL, 32611, Student Member AIAA

"Distinguished Professor, University of Florida, Gainesville, FL, 32611, Fellow AIAA

${ }^{\ddagger}$ Branch Chief, Propellant Delivery Fluids Branch, ER42, NASA/Marshall Space Flight Center, AL 35812

${ }^{\S}$ Flow Testing and Analysis Team, Propulsion Structural, Thermal, and Fluid Analysis Division Propulsion Systems Department Engineering, NASA/Marshall Space Flight Center, AL, 35812

${ }^{* *}$ Aerospace Engineer, NASA/Marshall Space Flight Center, AL 35812, Associate Fellow AIAA

${ }^{\dagger}$ Riverbend Design Services, Palm Beach Gardens, FL

"Clarence L. "Kelly" Johnson Collegiate Professor and Department Chair, University of Michigan, Ann Arbor, MI, 48109, Fellow AIAA 
from the combustion process with which to preheat the fuel, resulting in low chamber pressure and temperature. This can be an advantage because lower fuel temperatures can improve turbine reliability. However, the low chamber pressure means that the turbine work output is also limited.

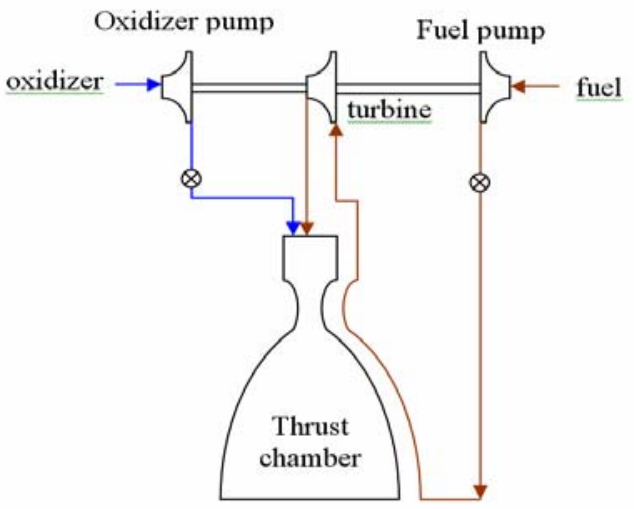

Figure 1. Expander cycle.

Turbine work can be increased in two ways: increasing the available energy in the drive gas or improving the efficiency by which the turbine can extract the available energy. Increasing the available energy for a given fluid is accomplished by increasing the turbine inlet temperature. To increase the turbine inlet temperature in an expander cycle, a higher heat flux from the thrust chamber to the cooling fuel is needed. Obtaining this higher heat flux is problematic in several ways. First, materials and manufacturing development is necessary to produce a thrust chamber with high heat flux capability. This work is an ongoing area of technology development. Second, to enable a higher heat exchange, increased surface area and contact time between the thrust chamber and cooling fuel is needed. These requirements lead to a larger, and heavier, thrust chamber. In addition, significantly raising the turbine inlet temperature defeats the expander cycle's advantage of maintaining a benign turbine environment. The second approach to increasing turbine work is to raise the turbine efficiency. If the turbine inlet temperature is held constant, an increase in turbine work is directly proportional to efficiency increase. If the required work can be achieved with moderate efficiency, an improvement in that efficiency can be traded for reduced inlet temperatures, providing better design environment margins.

One way to improve turbine efficiency is to use a radial turbine. Radial inflow turbines perform better than axial turbines at high velocity ratios, exhibit better tolerance to blade incidence changes, and have lower stresses than axial designs. Radial turbines have been used successfully in automotive applications ${ }^{2}$, but are not often used in rocket engines due to their relatively large size and weight. The size of compact radial turbines makes them applicable to rocket engine cycles when a high velocity ratio is involved.

The radial turbine design must provide maximum efficiency while keeping the overall weight of the turbine low. This necessitates a multi-objective optimization. A response surface analysis ${ }^{3}$ provides an efficient means of tackling the optimization problem. Response surfaces have been successfully used in prior rocket component design optimizations $^{6,7,8}$. Papila et al. performed a preliminary optimization of an axial turbine $e^{4,5}$ via a response surface analysis followed by a detailed shape design ${ }^{6,9}$ using response surface analysis and neural networks. Similarly, the research presented here represents the preliminary optimization of a radial turbine using a simplified 1-D radial turbine model adapted from the 1-D Meanline ${ }^{10}$ code utilized by Papila et al. The 1-D code is verified using 3-D simulations. Using response surface analysis, an accurate surrogate model will be constructed to predict the radial turbine weight and the efficiency across the selected design space. Because the radial turbine represents a new design, the feasible design space is initially unknown. Techniques including design constraint boundary identification and design space reduction are necessary to obtain an accurate response surface approximation (RSA).

\section{Problem Description}

The radial turbine performance was simulated using the 1-D Meanline ${ }^{10}$ code. Using a 1-D code allowed for the availability of relatively inexpensive computations. To determine whether using the 1-D code was feasible for 
optimization purposes, a 3-D verification study was conducted. Once the Meanline code was verified, the radial turbine optimization could proceed.

\section{A. Verification Study}

Three-dimensional unsteady Navier-Stokes simulations were performed for the baseline radial turbine design, and the predicted performance parameters were compared with the results of the Meanline analysis. Simulations were performed at three rotational speeds: the baseline rotational speed of 122,000 RPM, a low speed of 103,700 RPM and a high-speed of 140,300 RPM. A 2-vane/1-rotor model was used. The simulations were run with and without tip clearance, and the computational grids contained approximately 1.1 million grid points.

The PHANTOM code was used to perform the numerical simulations ${ }^{11}$. The governing equations in the PHANTOM code are the three-dimensional, unsteady, Navier-Stokes equations. The equations have been written in the Generalized Equation Set (GES) format ${ }^{12}$, enabling it to be used for both liquids and gases at operating conditions ranging from incompressible to supersonic flow. A modified Baldwin-Lomax turbulence model is used for turbulence closure ${ }^{13}$. In addition to the perfect gas approximation, the code contains two options for the fluid properties. The first option is based on the equations of state, thermodynamic departure functions, and corresponding state principles constructed by Oefelein ${ }^{14}$. The second option is based on splines generated from the NIST Tables ${ }^{15}$. A detailed description of the code/algorithm development, as well as its application to several turbine and pump test cases, is presented in Refs. 11 and 16.

Figure 2 shows static pressure contours (psi) at the mid-height of the turbine for the baseline rotational speed of 122,000 RPM. This figure illustrates the geometry of the turbine, and the contours indicate that the pressure decrease is nearly evenly divided between the vane and the rotor. In fact, the reaction was approximately 0.60 for each of the three 3-D simulations as compared to 0.55 for the 1-D simulation. Figure 3 contains the predicted totalto-static efficiencies from the Meanline and CFD analyses. The CFD results include values with and without tip clearance. In general, fair agreement is observed between the Meanline and CFD results. The trends are qualitatively similar, but the Meanline analysis predicts higher efficiencies. There is approximately a four-point difference in the quantitative values. The quantitative differences in the results are not surprising considering the lack of experimental data available to anchor the Meanline code. The differences in the predictions with and without tip clearance decrease with increasing rotational speed. Figure 4 shows the predicted work from the Meanline and CFD analyses. The trends are again similar between the Meanline and CFD analyses, but the Meanline values are consistently 5-6\% higher than the CFD values.

The similar trends between the 1-D Meanline code and 3-D CFD analyses indicate that the optimization can be confidently performed on the 1-D Meanline code. It can be expected that for a given turbine speed the Meanline code will over-predict the total-to-static efficiency by an expected degree. The predicted optimum point based on the 1-D Meanline code will likely yield overly optimistic results, but the predicted degree of improvement should translate to the 3-D CFD analysis.

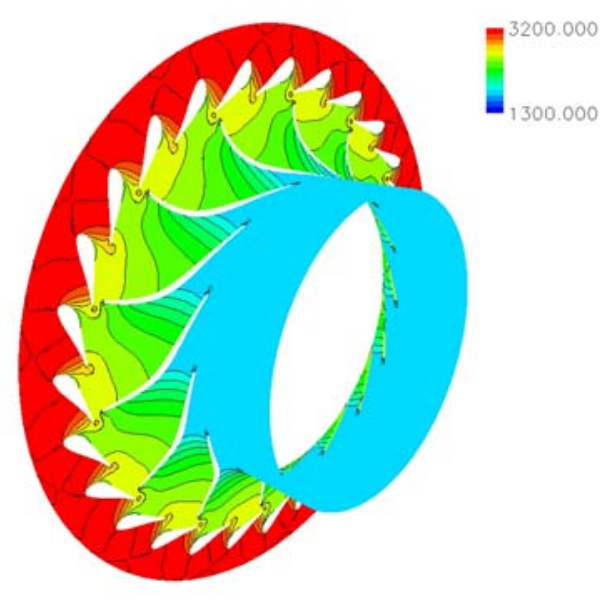

Figure 2. Mid-height static pressure (psi) contours at 122,000 rpm. 


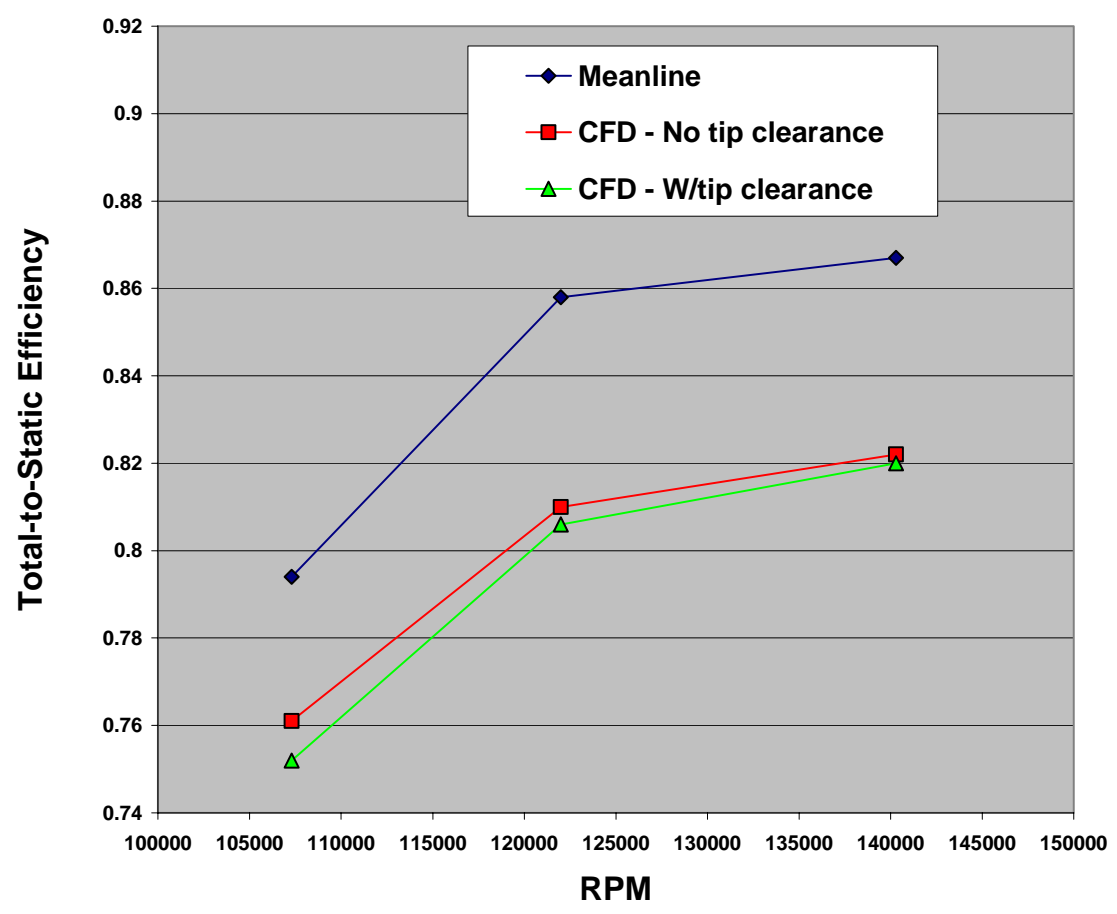

Figure 3. Predicted Meanline and CFD total-to-static efficiencies.

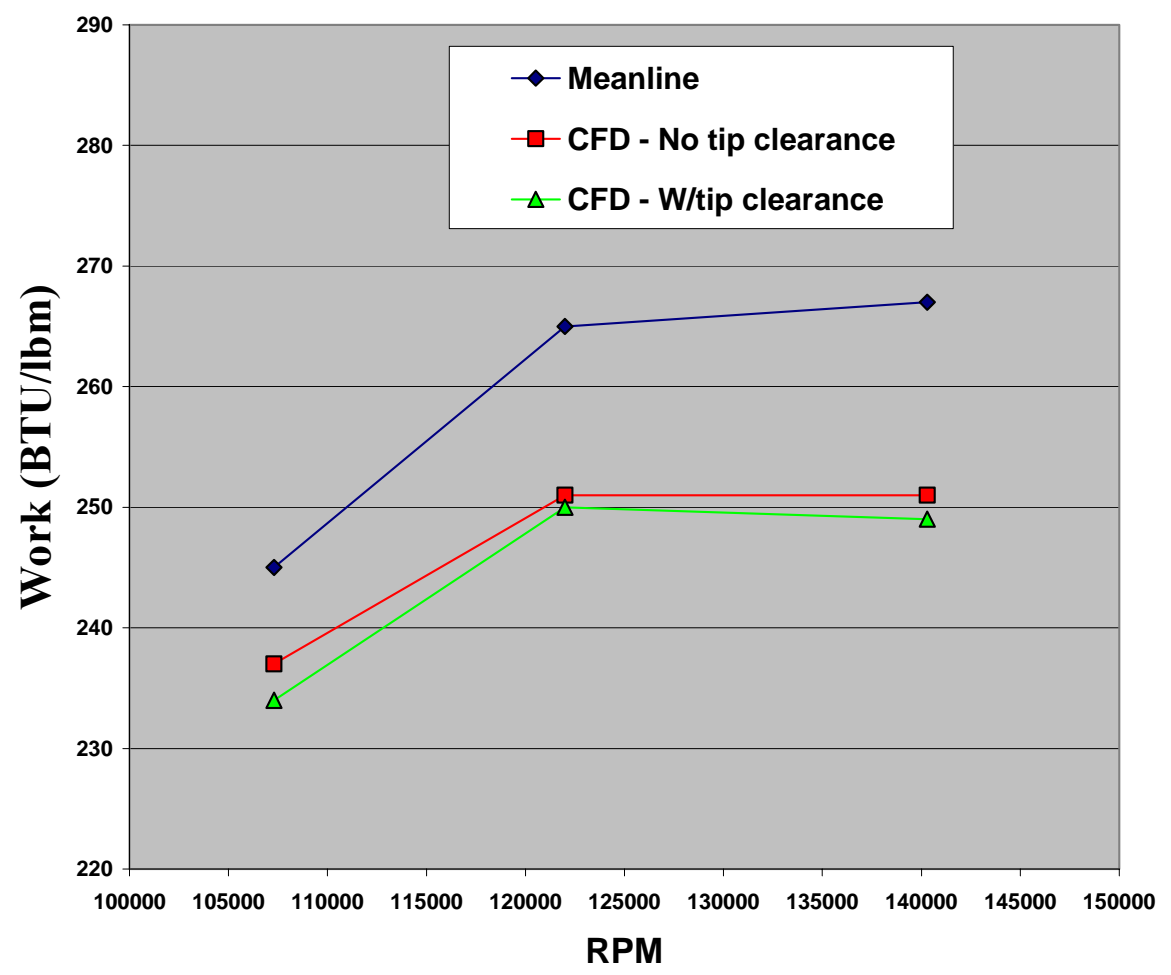

Figure 4. Predicted Meanline and CFD work.

American Institute of Aeronautics and Astronautics 


\section{B. Optimization Procedure}

The purpose of the design optimization is to maximize the turbine total-to-static efficiency while minimizing the turbine weight. A total of six design variables were identified as input variables to the Meanline code. The ranges of the design variables were set based on current radial turbine design practices. Additionally, five constraints were identified. In addition to the objective variables, the constraints are outputs from the Meanline code and are functions of the design variables. Two of the five constraints are structural constraints, two are geometric constraints, and one is an aerodynamic constraint. The aerodynamic constraints are based on general guidelines. The descriptions of all variables are given in Table 1. It was unknown in what way the constraints depended on the design variables, so it was possible that certain combinations of the design variables would cause constraint violations. It was also unknown whether the selected ranges would result in feasible designs. A response surface analysis was used to help clarify these unknown factors.

Table 1. Variable names and descriptions.

\begin{tabular}{|c|c|c|c|c|}
\hline Objective Variable & Description & \multicolumn{3}{|c|}{ Baseline design } \\
\hline$W_{\text {rotor }}$ & Relative measure of "goodness" for overall weight & \multicolumn{3}{|c|}{1.147} \\
\hline$\eta_{t s}$ & Total-to-static efficiency & \multicolumn{3}{|c|}{$85 \%$} \\
\hline Design Variable & & MIN & Baseline & MAX \\
\hline$R P M$ & Rotational Speed & 80,000 & 122,000 & 150,000 \\
\hline React & Percentage of stage pressure drop across rotor & 0.45 & 0.55 & 0.70 \\
\hline U/C isen & Isentropic velocity ratio & 0.50 & 0.61 & 0.65 \\
\hline Tip Flw & Ratio of flow parameter to a choked flow parameter & 0.30 & 0.25 & 0.48 \\
\hline Dhex\% & Exit hub diameter as a $\%$ of inlet diameter & 0.10 & 0.58 & 0.40 \\
\hline AnsqrFrac & Used to calculate annulus area (stress indicator) & 0.50 & 0.83 & 1.0 \\
\hline Constraints & & \multicolumn{3}{|c|}{ Desired Range } \\
\hline Tip Spd & Tip speed (ft/sec) (stress indicator) & \multicolumn{3}{|c|}{$\leq 2500$} \\
\hline$A N^{2}$ & Annulus area times speed squared (stress indicator) & \multicolumn{3}{|c|}{$\leq 850$} \\
\hline$\beta_{1}$ & Blade inlet flow angle & \multicolumn{3}{|c|}{$0 \leq \beta_{1} \leq 40$} \\
\hline Cx2/Utip & $\begin{array}{l}\text { Recirculation flow coefficient (indication of pumping } \\
\text { upstream) }\end{array}$ & \multicolumn{3}{|c|}{$\geq 0.20$} \\
\hline$R \operatorname{sex} / R \sin$ & $\begin{array}{l}\text { Ratio of the shroud radius at the exit to the shroud } \\
\text { radius at the inlet }\end{array}$ & \multicolumn{3}{|c|}{$\leq 0.85$} \\
\hline
\end{tabular}

The optimization problem is

Maximize $\eta_{t s}$ and Minimize $W_{\text {rotor }}$

such that

$$
\begin{aligned}
& 80000 \leq R P M \leq 150000 \\
& 0.45 \leq \text { React } \leq 0.70 \\
& 0.50 \leq \text { U/C isen } \leq 0.65 \\
& 0.30 \leq \text { Tip Flw } \leq 0.48 \\
& 0.10 \leq \text { Dhex } \% \leq 0.40 \\
& 0.50 \leq \text { AnsqrFrac } \leq 1.0
\end{aligned}
$$

In multi-objective optimizations the objectives often conflict, so that minimizing one objective function will increase the second objective function. A common method of dealing with conflicting objectives is by using a single composite objective function, such as a weighted sum or a desirability function. This approach will provide a single optimum point for each parameter setting in the composite function. However, in many cases, the desirability function or reasonable weights for the different objectives are not known before a solution is obtained. In this case, it is desirable to obtain a number of solutions that represent the best possible combination of objectives. If one 
function is plotted versus the other function, the best possible solutions will be represented by a curve that leads from the optimum of one function to the optimum of the second function. The points along the curve will be the best "trade-off" solutions. In mathematical terms, the curve represents a set of non-dominated points called the Paretooptimal set.

In this study, an elitist non-dominated sorting genetic algorithm NSGA-II ${ }^{17}$ with a parallel archiving strategy to overcome the Pareto drift problem ${ }^{19}$ is used as the multi-objective optimizer to generate Pareto optimal solutions. The description of the algorithm is given as follows:

1. Randomly initialize a population (designs in the design space) of size nрop.

2. Compute objectives and constraints for each design.

3. Rank the population using non-domination criteria. Many individuals can have the same rank with the best individuals given the designation of rank-1. Initialize an archive with all the non-dominated solutions.

4. Compute the crowding distance. This distance finds the relative closeness of a solution to other solutions in the function space and is used to differentiate between the solutions on same rank.

5. Employ genetic operators - selection, crossover, and mutation — to create intermediate population of size прор.

6. Evaluate objectives and constraints for this intermediate population.

7. Combine the two (parent and intermediate) populations, rank them, and compute the crowding distance.

8. Update the archive:

- Compare archive solutions with rank-1 solutions in the combined population.

- Remove all dominated solutions from the archive.

- Add all rank-1 solutions in the current population which are non-dominated with respect to the archive.

9. Select a new population nрор from the best individuals based on the ranks and the crowding distances.

10. Go to step 3 and repeat until the termination criteria is reached, which in the current study is chosen to be the number of generations.

Other popular methods of constructing the Pareto-optimal set can be found in Knowles et al. ${ }^{20}$ and contained references.

\section{Response Surface and Global Sensitivity Analysis Techniques}

\section{A. Response Surface Methodology}

The response surface method assumes that the system response $y$ can be expressed as a function of its design variables $\xi_{1}, \xi_{2}, \ldots, \xi_{N}$ plus an error term $\varepsilon$ that encompasses effects such as measurement error, numerical error, and error from background noise:

$$
y=f\left(\xi_{1}, \xi_{2}, \ldots, \xi_{N}\right)+\varepsilon
$$

The response surface method usually assumes a polynomial approximation for $f(\xi)+\varepsilon$. Thus, the approximated response can now be written in terms of the scaled variables $x_{1}, x_{2}, \ldots, x_{N}$ as

$$
\hat{y}=f\left(x_{1}, x_{2}, \ldots, x_{N}\right)
$$

For $N$ design variables, the first order model is given as

$$
\hat{y}=\beta_{0}+\beta_{1} x_{1}+\beta_{2} x_{2}+\cdots+\beta_{N} x_{N}
$$

and the second order model is given as

$$
\hat{y}=\beta_{0}+\sum_{j=1}^{N} \beta_{j} x_{j}+\sum_{j=1}^{N} \beta_{j j} x_{j}^{2}+\sum \sum_{i<j=2}^{N} \beta_{i j} x_{i} x_{j}
$$


The pattern can be continued for third-order models, etc.

The response surface parameters $\beta_{0}, \beta_{j}, \beta_{i j}$, and $\beta_{i j}$ can be determined using the method of least squares. These coefficients are chosen via least squares regression to minimize the sum of the squares of the errors between the approximated response and the actual response. The function to be minimized is called the loss function, and the least squares loss function is given by

$$
L=\sum_{i=1}^{n}\left(y_{i}-\hat{y}_{i}\right)^{2}
$$

where $n$ is the number of design points. A more general form of the loss function allows some flexibility in the selection of a surrogate model. The general form is given by

$$
L=\sum_{i=1}^{n}\left|y_{i}-\hat{y}_{i}\right|^{p}
$$

where $p=2$ for least square regression.

In matrix notation, the response is given as

$$
\mathbf{y}=\mathbf{X} \boldsymbol{\beta}
$$

The matrix $\boldsymbol{\beta}$ can then be determined using the equation

$$
\boldsymbol{\beta}=\left(\mathbf{X}^{\prime} \mathbf{X}\right)^{-1} \mathbf{X}^{\prime} \mathbf{y}
$$

The first measure of the quality of a response surface is the coefficient of multiple determination, $R^{2}$. This value describes the amount of reduction in the variability of the response using the regressor variables. In the case of least squares regression, it is equal to one minus the sum of the squares of the errors $\left(S S_{E}\right)$ divided by the total sum of squares $\left(S S_{T}\right)$ :

$$
R^{2}=1-\frac{S S_{E}}{S S_{T}}
$$

where

$$
\begin{gathered}
S S_{E}=\sum_{i=1}^{n}\left(y_{i}-\hat{y}_{i}\right)^{2} \\
S S_{T}=\sum_{i=1}^{n} y_{i}^{2}-\frac{1}{n}\left(\sum_{i=1}^{n} y_{i}\right)^{2}
\end{gathered}
$$

and $n$ is the total number of points. An estimate of the standard deviation of the error in the response surface is the root mean square (RMS) error. The RMS error is a second measure of response surface quality and is given by

$$
\hat{\sigma} \approx R M S \text { error }=\sqrt{\frac{S S_{E}}{n-m}}
$$

where $m$ is the number of terms in the function approximation.

Based on Eq.(10), it can be seen that the range of $R^{2}$ is $0 \leq R^{2} \leq 1$. A value close to one implies a good fit. However, this equation does not take into account the number of terms in the approximating function. Adding terms to the function approximation will always increase the value of $R^{2}$, but will not necessarily improve the function's 
prediction capability. In this case, it is advantageous to use the adjusted coefficient of multiple determination, $R_{\text {adj. }}^{2}$. This measure of fit accounts for the number of terms by penalizing against nonessential terms. It is given by

$$
R_{a d j}^{2}=1-\left(\frac{n-1}{n-m}\right)\left(1-R^{2}\right)
$$

\section{B. Design of Experiments}

Data points were initially selected based on a face-centered central composite design of experiments ${ }^{3}$ (DOE) for a total of 77 data points within the selected design variable ranges. The face-centered central composite design uses the corners and center faces of an $\mathrm{N}$-dimensional cube for point selection. Other DOEs used in the process of refining the range of interest include Latin Hypercube Sampling ${ }^{17}$ and the factorial design ${ }^{3}$. Latin-hypercube is a stratified sampling technique where the ranges of the design variables are divided into either equal intervals or intervals reflecting a distribution function, and a point is randomly selected from each interval. This insures that the points selected cover the entire design space. Full factorial designs distribute points uniformly in design space.

\section{Global Sensitivity Analysis}

Unlike local sensitivity where the partial derivatives are used to locally estimate the sensitivity of an objective to a specific design variable, global sensitivity allows the study of overall model behavior and is a measure of the contribution of an independent variable to the total variance of the dependent data. Global sensitivity analyses allow a variety of issues to be addressed. Of particular interest in the context of the present study are

1. Variables prioritization, i.e., ranking a set of design variables according to their contribution to the output variability

2. Evidence or absence of parameter interactions

3. Regions of interest in the parameter space if additional samples become available

4. Model validation, i.e., whether the model reproduces well known behavior of the process of interest

A detailed discussion of global sensitivity methods and applications can be found in Sobol ${ }^{21}$, Homma and Saltelli ${ }^{22}$, Saltelli et al., ${ }^{23}$ and the references therein.

A surrogate model $f(\mathbf{x})$ of a square integrable objective as a function of a vector of independent design variables, $\mathbf{x}$, is assumed and modeled as uniformly distributed random variables. The surrogate model as given in Eq. (5) can be decomposed as the sum of functions of increasing dimensionality as

$$
f(\mathbf{x})=f_{0}+\sum_{i} f_{i}\left(x_{i}\right)+\sum_{i<j} f_{i j}\left(x_{i}, x_{j}\right)+\cdots+f_{12 \ldots N}\left(x_{1}, x_{2}, \ldots, x_{N}\right)
$$

where

$$
f_{0}=\int_{\mathbf{x}=0}^{1} f \mathrm{~d} \mathbf{x}
$$

If the following condition

$$
\int_{0}^{1} f_{i_{1} \ldots i_{s}} d x_{k}=0
$$

is imposed for $k=i_{1}, \ldots, i_{s}$, then the decomposition described in Eq. (15) is unique.

In this context, the total variance denoted as $V(f)$ can be shown to be equal to

$$
V(f)=\sum_{i=1}^{n} V_{i}+\sum_{1 \leq i \leq j \leq N} V_{i j}+\cdots+V_{1 \ldots N}
$$


where each of the terms in Eq. (17) represents the partial contribution, or partial variance, of the independent variables $\left(V_{i}\right)$, or set of variables to the total variance, and provides an indication of their relative importance. The partial variances can be calculated using the following expressions:

$$
\begin{aligned}
& V_{i}=V\left(E\left[f \mid x_{i}\right]\right) \\
& V_{i j}=V\left(E\left[f \mid x_{i}, x_{j}\right]\right)-V_{i}-V_{j}
\end{aligned}
$$

and so on, where $\mathrm{V}$ and $\mathrm{E}$ denote variance and the expected value respectively. Note that $E\left[f \mid x_{i}\right]=\int_{0}^{1} f_{i} d x_{i}$ and $V\left(E\left[f \mid x_{i}\right]\right)=\int_{0}^{1} f_{i}^{2} d x_{i}$. All integrals were calculated by direct integration of Eq. (5) as a function of x. Now the sensitivity indices can be computed corresponding to the independent variables and set of variables. For example, the first and second order sensitivity indices can be computed as

$$
S_{i}=\frac{V_{i}}{V(f)}, \quad S_{i j}=\frac{V_{i j}}{V(f)}
$$

Under the independent model inputs assumption, the sum of the sensitivity indices is equal to one.

\section{Results and Discussion}

\section{Phase 1: Initial Design of Experiments and Constraint Violations}

The values of the objective functions were obtained using the Meanline ${ }^{10}$ code. Of the 77 solutions from the initial DOE, seven cases failed and 60 cases violated one or more of the five constraints, resulting in only 10 successful cases. Before the optimization could be conducted, a feasible design space needed to be identified. Because there was limited information on the dependencies of the output constraints on the design variables, response surface analyses were used to determine these dependencies. Response surfaces were used to properly scale the design variable ranges and identify irregular constraint boundaries.

Response surfaces were fit to the output constraints. The change in each response surface with respect to changes in some of design variables was small, indicating that the response surfaces were largely insensitive to certain design variables. The accuracy of the response surfaces was not compromised when the identified design variables were neglected. Therefore, for each response surface, the effects of variables that contributed little to the response surface were removed. In this way, each response surface was simplified. The simplified dependencies are shown in Eq. (20).

$$
\begin{aligned}
& A N^{2}=A N^{2}(\text { AnsqrFrac }) \\
& \text { Tip Spd }=\text { Tip Spd }(\text { U/C isen }) \\
& \text { Cx2/Utip }=\text { Cx2/Utip }(\text { RPM }, \text { U/C isen, AnsqrFrac }) \\
& \beta_{1}=\beta_{1}(\text { React }, \text { U/C isen,Tip Flw }) \\
& \text { Rsex/Rsin }=\text { Rsex/Rsin (AnsqrFrac,U/C isen,Dhex\%) }
\end{aligned}
$$

The information obtained from the response surfaces about the output constraint dependences were further used to develop constraints on the design variables. A response surface was constructed for each design variable as a function of the output constraint and the remaining design variables, for example

$$
\text { React }=\operatorname{React}\left(\beta_{1}, \text { U/Cisen,Tip Flw }\right)
$$

The most accurate response surfaces $\left(R_{a d j}^{2} \geq 0.99\right)$ were used to determine the design variable constraints. The output constraints were in turn set to the constraint limits. For example, a constraint on React was applied to coincide with the constraint $\beta_{1} \geq 0$ : 


$$
\begin{aligned}
& \beta_{1}=\beta_{1}(\text { React }, \text { U/Cisen,Tip Flw }) \geq 0 \\
& \Rightarrow \operatorname{React} \leq \operatorname{React}\left(\beta_{1}=0, \text { U/Cisen,Tip Flw }\right)
\end{aligned}
$$

Constraint boundary approximations were developed in this manner for each constraint. The equations used to define each constraint are given in Table A of the Appendix. As can be seen from Eq.(20), two of the five constraints $\left(A N^{2}\right.$ and Tip Spd) were simple limits on a single variable. For the single variable constraints, the variable ranges were simply reduced to match the constraint boundaries. The remaining constraints were more complex. However, one of the complex constraints $(R \operatorname{sex} / R \sin )$ was automatically satisfied by the reduction of the variable ranges for the single variable constraints. The feasible region is shown in Figure 5.

The remaining constraints involved three variables each. It was discovered that many low values of $R P M$ violated the Cx2/Utip constraint. The region of violation was a function of $R P M, U / C$ isen, and AnsqrFrac as shown in Figure 6. The $\beta_{l}$ constraint was found to be the most demanding and resulted in a feasible design space as shown in Figure 7. Much of the original design space violated this constraint. It was also discovered that the constraint surface representing the bounds for $\beta_{1} \geq 40$ lay outside of the original design variable range for React. In this case, the lower bound for React was sufficient to satisfy this constraint.

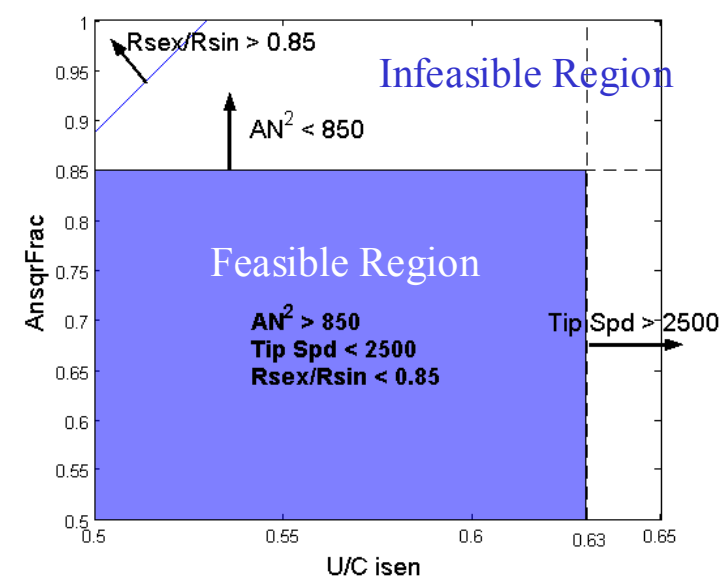

Figure 5. Constraint regions for three constraints. Three of five constraints are automatically satisfied by the range reduction of two design variables.

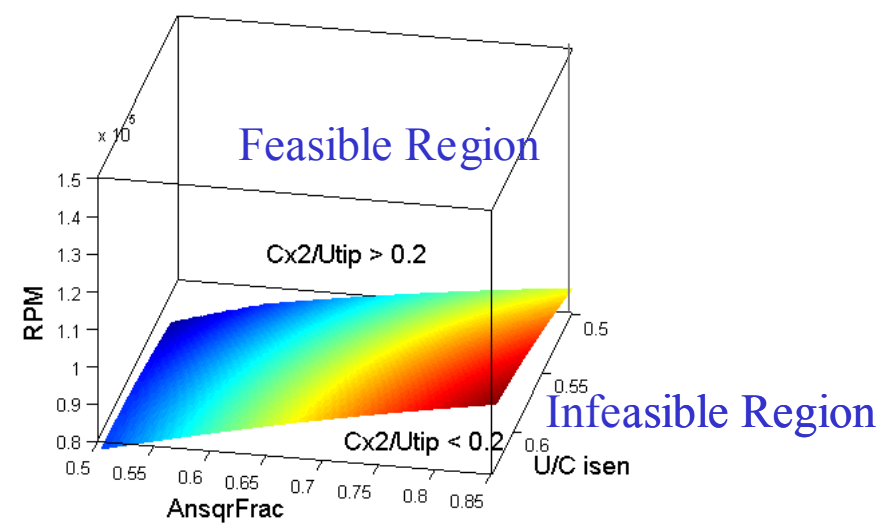

Figure 6. Constraint surface for $C x 2 /$ Utip $=0.2$. At higher values of AnsqrFrac and $U / C$ isen, lower values of $R P M$ are invalid. 

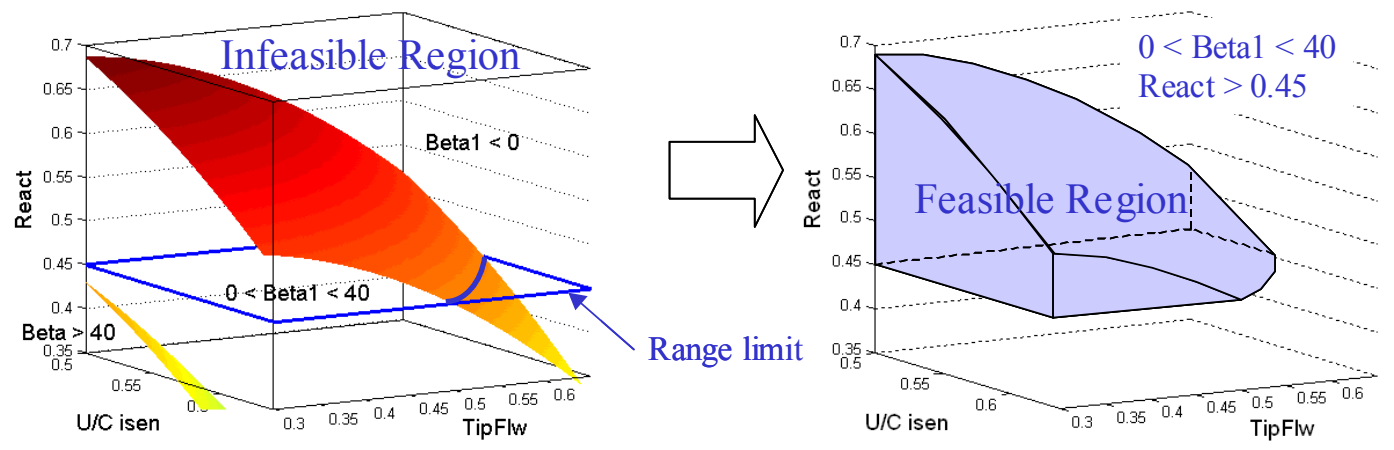

Figure 7. Constraint surfaces for $\beta_{1}=0$ and $\beta_{1}=40$. Values of $\beta_{1}>40$ lay outside of design variable ranges.

The predictive capability of the constraints was tested using the available data set. The design variable values were input into the RSAs for the constraints. Using the RSAs, all points that violated the output constraints were correctly identified. Now that the feasible design space was accurately identified, data points could be placed in the feasible data region. The results and summary of the prediction of constraint violations are as follows:

1. Response surfaces were constructed to determine the relationship between the output constraints and the design variables.

2. The variable ranges were adjusted based on information from the constraint surfaces.

3. A 3-level factorial design (729 points) was applied within new variable ranges.

4. Points that violated the constraints (498 / 729 points) were eliminated based on RSAs of constraints.

Using the response surface constraint approximations, $97 \%$ of the 231 new data points predicted to be feasible lay within the actual feasible design space region after simulation using the Meanline code. The points that were predicted to be feasible, but were actually found to be infeasible, often violated the $\beta_{1} \geq 0$ requirement by a slight amount.

\section{Phase 2: Design Space Refinement}

Plotting the data points in function space revealed additional information about the location of the design space as shown in Figure 8. A large area of function space contained data points with a lower efficiency than was desired. There also existed areas of high weight without improvement in efficiency. Additionally, the fidelity of the response surface for $\eta_{t s}$ was apparently compromised by the existence of a design space that was too large as evidenced by the fit characteristics given in Table B of the Appendix. These undesirable areas could be eliminated, and the response surface fidelity could be improved by refining the design space. The density of points could then be increased within the region of interest, eliminating the possibility of unnecessary investigation of undesirable points. The region of interest is shown in Figure 8.

To further test the necessity of a reduced design space, five RSAs each were used to fit the data for $W_{\text {rotor }}$ and $\eta_{t s}$ in the original feasible design space. These response surfaces were constructed using the general loss function given in Eq. (7) for $p=1 \ldots 5$. The RSA constructed using the least square loss function $(p=2)$ was used as a reference point. As seen in Figure 9, regardless of the RSA used, the error in the RSA at the data points is high at high $W_{\text {rotor }}$ and low $\eta_{t s}$. To improve RSA performance, additional data points could be added in these regions, or these data regions could be eliminated.

Pareto fronts were constructed for each RSA set. The results are shown in Figure 10. In this case, the Pareto fronts differ by as much as $20 \%$. Because the results differ significantly depending on which RSA is used, this also indicates that further design space refinement is necessary. 


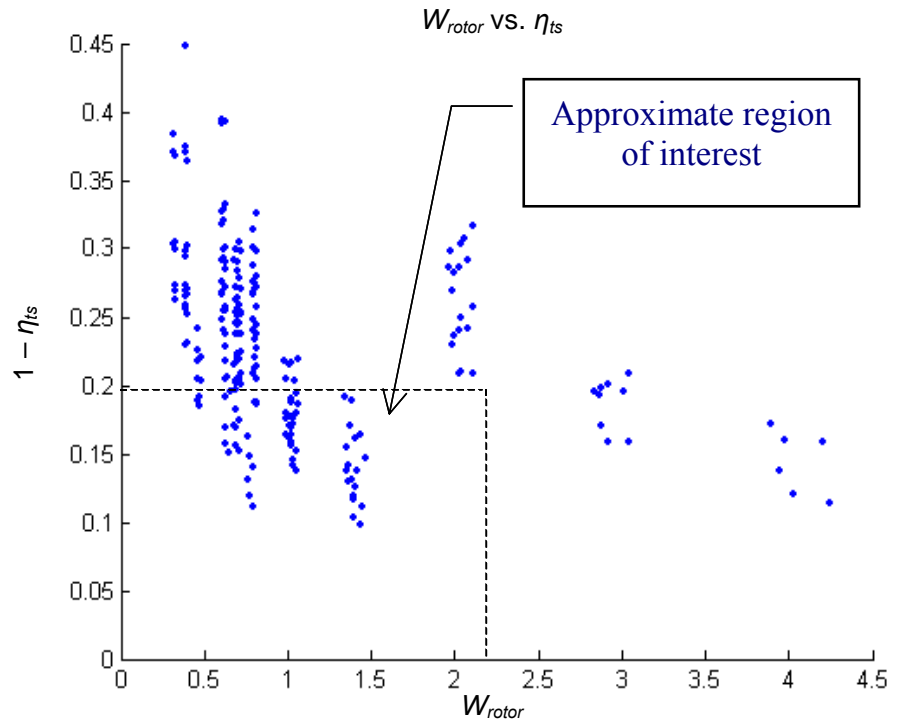

Figure 8. Region of interest in function space. (The quantity $1-\eta_{t s}$ is used for improved plot readability.)
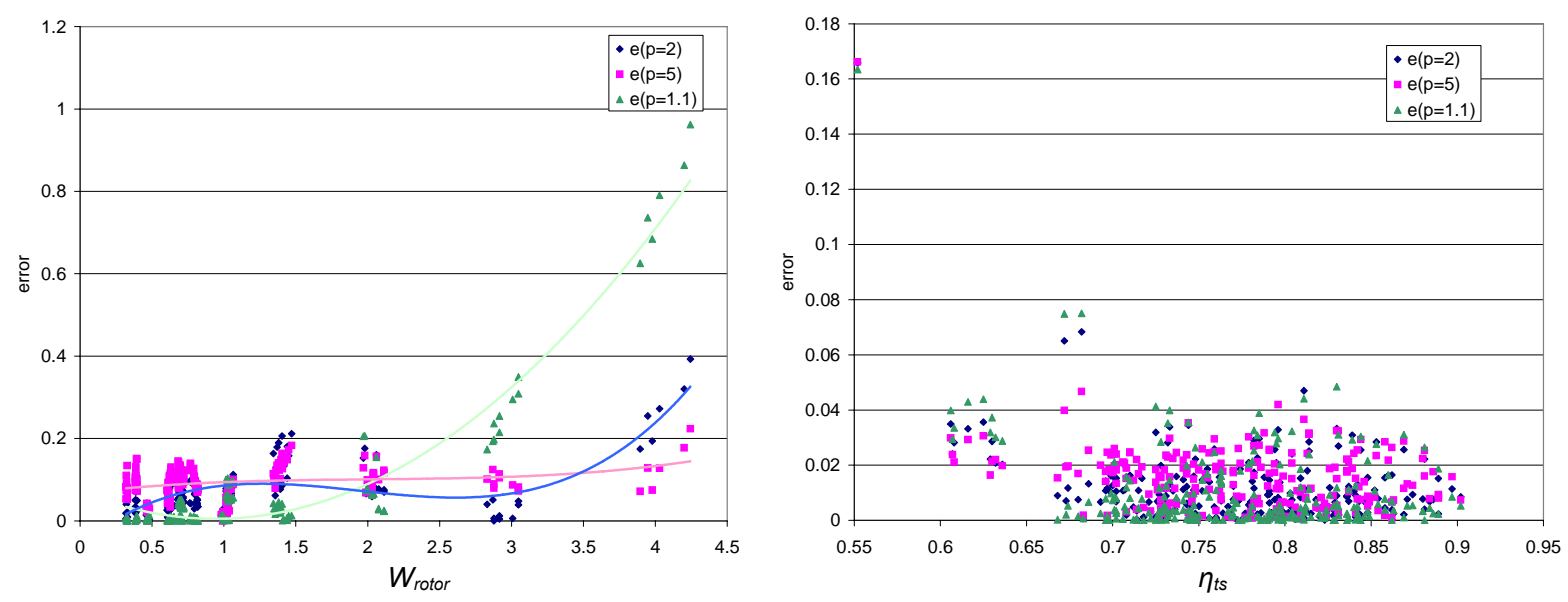

Figure 9. Error between RSA and actual data point for $p=1.1,2$, and 5. 


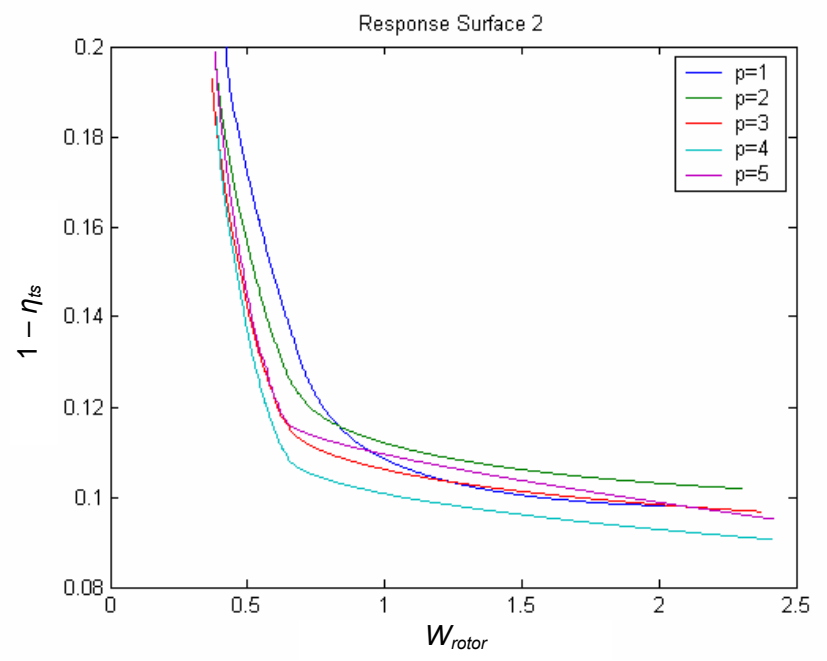

Figure 10. Pareto fronts for $p=1 \ldots .5$ for second data set. (The quantity $1-\eta_{t s}$ is used for improved plot readability.) Pareto fronts differ by as much as $20 \%$.

The design variable bounds were further reduced to match the new design space. The new design variable ranges are given in Table 2. Response surfaces were constructed for the turbine weight, $W_{\text {rotor }}$, and the turbine total-to-static efficiency, $\eta_{t s}$, using the original feasible design space to screen data points. Points predicted to lie outside of the new design space would be omitted. For the refined design space, a third set of data was required. As seen in Figure 8 , using a factorial design tended to leave holes in function space. It was possible that this could hamper construction of an accurate Pareto front. To prevent this, Latin Hypercube Sampling was the DOE used for the third data set. The points were efficiently distributed by maximizing the minimum distance between any points. By evaluating the best points from the second data set, it was determined that only RPM, Tip Flw, and U/C isen varied among these points. To ensure that this effect was captured in the third data set, additional points were added using a 5-level factorial design over these three variables. Least square response surfaces previously constructed for the turbine weight, $W_{\text {rotor }}$, and the turbine total to static efficiency, $\eta_{t s}$, were used to screen the potential data points. Points predicted to lie outside of the new design space would be omitted from the analysis. In summary,

1. Only the portion of design space with best performance was reserved to allow for a concentrated effort on the region of interest and to increase response surface fidelity.

2. Latin Hypercube Sampling was used over all six variables and was supplemented by a 5-level factorial design used over RPM, Tip Flw, and U/C isen.

3. Points that were predicted to violate constraints or lie outside of region of interest were omitted. The combination of the DOEs resulted in a total of 323 feasible design points.

Table 2. Original and final design variable ranges after constraint application and design space reduction.

\begin{tabular}{llcccc}
\hline $\begin{array}{l}\text { Design } \\
\text { Variable }\end{array}$ & Description & $\begin{array}{c}\text { MIN } \\
\text { Original }\end{array}$ & $\begin{array}{c}\text { MAX } \\
\text { Range }\end{array}$ & \multicolumn{2}{c}{$\begin{array}{c}\text { MIN } \\
\text { Final Ranges }\end{array}$} \\
\hline \hline RPM & Rotational Speed & 80,000 & 150,000 & 100000 & 150,000 \\
React & Percentage of stage pressure drop across & 0.45 & 0.68 & 0.40 & 0.57 \\
U/C isen & rotor & 0.5 & 0.63 & 0.56 & 0.63 \\
Tip Flw & $\begin{array}{l}\text { Ratio of flow parameter to a choked flow } \\
\text { parameter }\end{array}$ & 0.3 & 0.65 & 0.3 & 0.53 \\
AnsqurFrac & Exit hub diameter as a \% of inlet diameter & 0.1 & 0.4 & 0.1 & 0.4 \\
& $\begin{array}{l}\text { Used to calculate annulus area (stress } \\
\text { indicator) }\end{array}$ & 0.5 & 0.85 & 0.68 & 0.85 \\
\hline
\end{tabular}




\section{Phase 3: Construction of the Pareto Front and Validation of Response Surfaces}

As in the second data set, five RSAs each with $p=1 \ldots 5$ were used to fit the data for $W_{\text {rotor }}$ and $\eta_{t s}$ for the third data set. Pareto fronts were constructed for each RSA set and are shown in Figure 11. In this case, the Pareto fronts differed by a maximum of only 5\%. Because the difference in the response surfaces for varying values of $p$ is small for the third data set, the design space was determined to be adequately refined.

Function evaluations from the least square response surfaces $(p=2)$ were used to construct the Pareto Front shown in Figure 12. Within the Pareto front, a region was identified that would provide the best value in terms of maximizing efficiency and minimizing weight. This trade-off region was selected for the validation of the Pareto front. The results of the subsequent validation simulations indicated that the response surfaces and corresponding Pareto front were very accurate. A noticeable improvement was attained compared to the baseline radial turbine design. The design selected had the same weight $\left(W_{\text {rotor }}\right)$ as the baseline case with approximately $5 \%$ improvement in efficiency. The specifications for the optimum design are given in Table 3.

Table 3. Baseline and optimum design comparison.

\begin{tabular}{llcc}
\hline Objectives & \multicolumn{1}{c}{ Description } & Baseline & Optimum \\
\hline \hline$W_{\text {rotor }}$ & Relative measure of "goodness" for overall weight & 1.147 & 1.147 \\
$\eta_{t s}$ & Total-to-static efficiency & $85.0 \%$ & $89.7 \%$ \\
\hline Design Variables & & Baseline & Optimum \\
\hline \hline$R P M$ & Rotational Speed & 122,000 & 124,500 \\
React & Percentage of stage pressure drop across rotor & 0.55 & 0.45 \\
U/C isen & Isentropic velocity ratio & 0.61 & 0.63 \\
Tip Flw & Ratio of flow parameter to a choked flow parameter & 0.25 & 0.30 \\
Dhex $\%$ & Exit hub diameter as a \% of inlet diameter & 0.58 & 0.10 \\
AnsqrFrac & Used to calculate annulus area (stress indicator) & 0.83 & 0.85 \\
\hline
\end{tabular}

Within the best trade-off region, only RPM and Tip Flw vary along the Pareto front as seen in Figure 13. The other variables are constant within the trade-off region and are set to their maximum or minimum value. This indicates that increasing the range of one of these variables might result in an increase in performance. The minimum value of the variable React was chosen as the only variable range that could reasonably be adjusted. The validation points were simulated again using a reduced React value. Reducing the minimum value of React from 0.45 to 0.40 increased the maximum efficiency only for $W_{\text {rotor }}>1$. The maximum increase in efficiency improved from $4.7 \%$ to $6.5 \%$, but this increase occurred outside of the preferred trade-off region. It was further determined that using a React value of 0.40 resulted in a turbine design that was too extreme.

Based on the results of the current study, several observations can be made concerning the design of highperformance radial turbines. The trends of the trade-off designs are shown in Figure 13.

1. The more efficient designs have a higher velocity ratio based on the rotational speed as shown by $U / C$ isen in Figure 13. This is a result of the inlet blade angle being fixed at 0.0 degrees due to structural considerations. Classic radial turbine designs have velocity ratios of approximately 0.70 .

2. The more efficient designs have a smaller tip radius and larger blades (AnsqrFrac) as compared to designs with a larger radius and smaller blades. Increasing the annulus area leads to higher efficiencies. In this case, the annulus area (AnsqrFrac) is set to the highest value allowable while respecting stress limitations.

3. The higher performing designs have higher rotational speeds (as compared to the original minimum $R P M$ value of 80,000 given in Table 1) as a result of the smaller radius and large blades. The higher rotational speeds also lead to more efficient pump operation. 


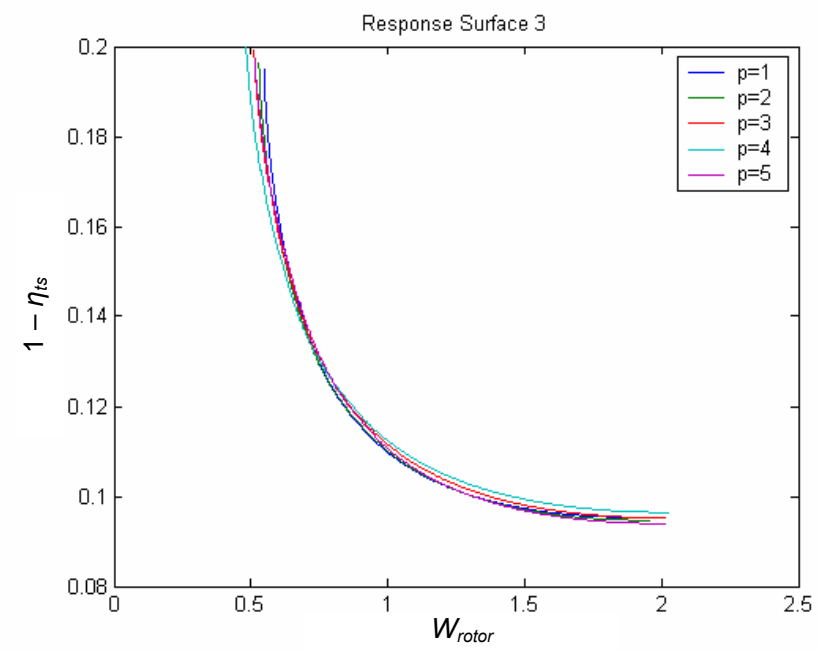

Figure 11. Pareto fronts for $p=1 \ldots 5$ for third data set. (The quantity $1-\eta_{t s}$ is used for improved plot readability.) Pareto fronts differ by no more than $5 \%$.

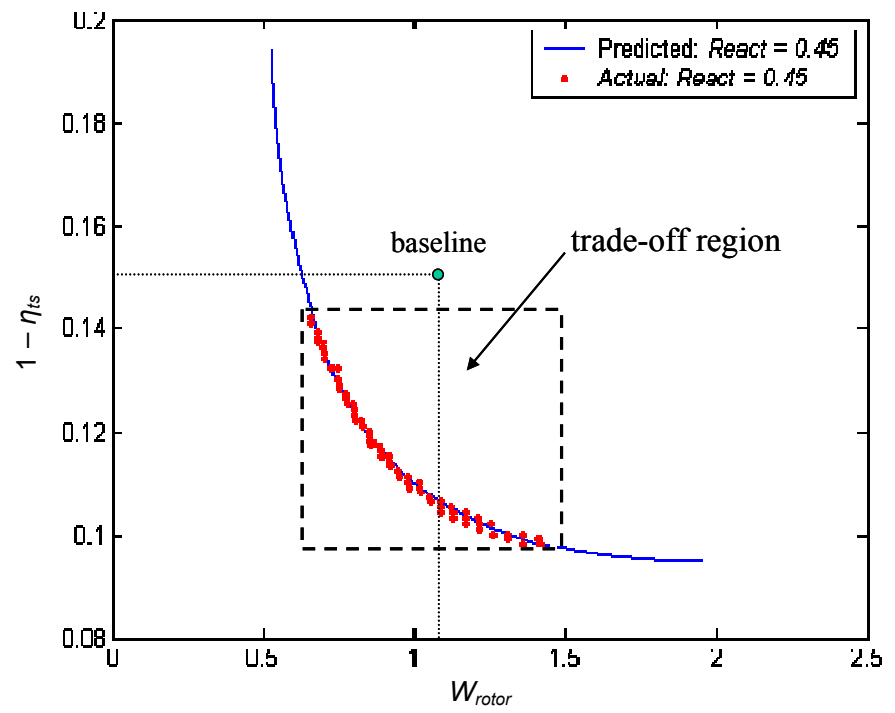

Figure 12. Pareto Front with validation data. Deviations from the predictions are due to rounded values of the design variables (predicted quantities use more significant digits). The quantity $1-\eta_{t s}$ is used for improved plot readability. 

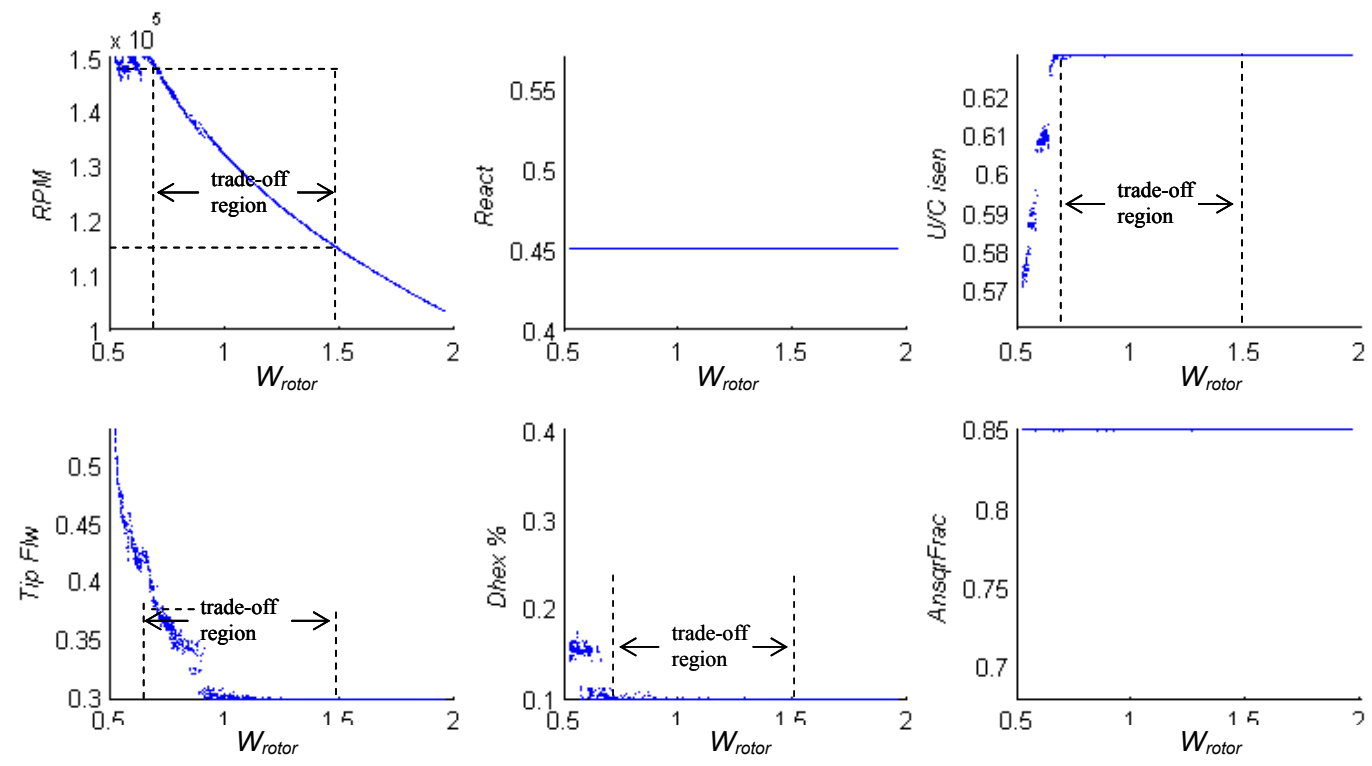

Figure 13. Variation in design variables along Pareto Front.

\section{Phase 4: Global Sensitivity Analysis}

A global sensitivity analysis was conducted using the response surface approximations for the final design variable ranges given in Table 3. The results are shown in Figure 14. It was discovered that the turbine rotational speed $R P M$ had the largest impact on the variability of the resulting turbine weight $W_{\text {rotor }}$. The effects of the rotational speed $R P M$ along with the isentropic velocity ratio $U / C$ isen make up $97 \%$ of the variability in $W_{\text {rotor }}$. All other variables and variable interactions have minimal effect on $W_{\text {rotor }}$. For the total to static efficiency, $\eta_{t s}$, the effects of the design variables are more evenly distributed. The reaction variable, React, has the highest overall impact on $\eta_{t s}$ at $28 \%$. This information is useful for future designers. For future designs, it may be possible to eliminate all variables except $R P M$ and $U / C$ isen when evaluating $W_{\text {rotor }}$, while for $\eta_{t s}$ it may be necessary to keep all variables.

It must be noted that the local variability at any given point can vary significantly from the global sensitivity values due to the nonlinear nature of the RSAs. However, the global sensitivity analysis can be scaled to the region of interest to explore more localized effects.

$$
W_{\text {rotor }}
$$

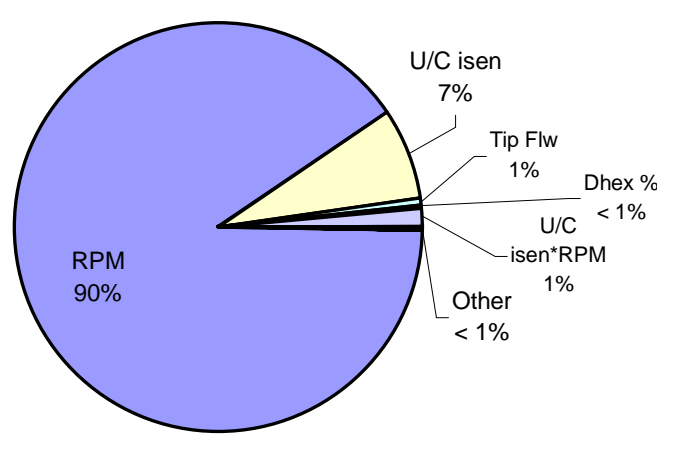

$\eta_{t s}$

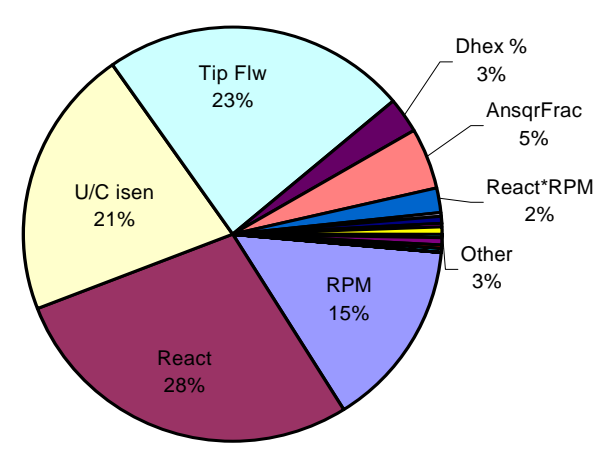

a) b)

Figure 14. Global sensitivity analysis. Effect of design variables on (a) $\boldsymbol{W}_{\text {rotor }}$ and (b) $\eta_{t s}$. 


\section{Conclusion}

The radial turbine optimization process began without a clear idea of the location of the feasible design region. Response surfaces based on output constraints were successfully used to identify feasible design space. The new design space was found to be too large to accommodate the construction of accurate response surface approximations for the prediction of turbine total-to-static efficiency. Reduction of the design space eliminated poorly performing areas while improving response surface fidelity. Using the Pareto front information, a best tradeoff region was identified within which the Pareto front was validated. In general, the best designs had high velocity ratios, large blades to extract the maximum energy from the flow, and high rotational speeds as a result of the small radius and large blades. A global sensitivity analysis provided a summary of the effects of design variables on objective variables. The rotational speed had the highest impact on the turbine weight, while all variables investigated had a significant impact on the efficiency. At the same weight, the RSA optimization resulted in approximately $5 \%$ improvement in efficiency over the baseline case.

Future work will include a comparison of the 1-D Meanline code results of the selected optimum case with a full 3-D simulation to determine if the efficiency gains are comparable over the baseline case. The next step in the radial turbine design process will be to conduct a blade shape optimization.

\section{Appendix: Details and Statistics on Selected Response Surface Approximations}

Supplemental information on various response surface approximations are given in Table A and Table B. Table A lists the specific response surface approximations for the variable constraints that are used throughout the analysis. The constraint approximations limit the number of infeasible points computed by the Meanline code. The methodology for obtaining the approximations is given in Phase 1 of Section IV. Table B details the statistics of the response surface approximations for the objective functions before and after reduction of the design space with design variable ranges given in Table 2. The various methods of measuring the accuracy of fit for RSAs are given in Section III.A. Before reduction of the design space, $\mathrm{R}^{2}$ and $\mathrm{R}_{\text {adj }}^{2}$ were low for the total-to-static efficiency $\boldsymbol{\eta}_{t s}$. Considerable improvement was attained after the design space was reduced, resulting in lower RMS error and $\mathrm{R}^{2}$ and $\mathrm{R}_{\text {adj }}^{2}$ values approximately equal to unity.

Table A. Specified output constraints with corresponding design variable constraints

\begin{tabular}{ll}
\hline Output Constraint & Design Variable Constraint Approximation \\
\hline \hline AN $N^{2} \leq 850$ & AnsqrFrac $\leq 0.85$ \\
Tip $S p d \leq 2500$ & U/C isen $\leq 0.637$ \\
Cx $2 / U t i p \geq 0.2$ & $R P M \geq-35364+270000 U+34282 A-258149 U^{2}+12470 A U-38090 A^{2}$ \\
$\beta_{1} \geq 0$ & React $\leq 0.234+2.28 U+0.745 T-3.04 U^{2}-0.356 T U-1.11 T^{2}$ \\
Rsex $/$ Rsin $\leq 0.85$ & AnsqrFract $\leq-0.291+0.487 U+2.47 D+4.78 U^{2}-4.49 D U-2.19 D^{2}$ \\
\hline where $U \equiv U / C$ isen, $T \equiv$ Tip Flw, D Dhex $\%, A \equiv$ AnsqrFrac
\end{tabular}

Table B. Response surface fit statistics before and after design space reduction.

\begin{tabular}{lcccc}
\hline & $\begin{array}{c}\boldsymbol{W}_{\text {rotor }} \\
\text { Before design space reduction }\end{array}$ & $\begin{array}{c}\boldsymbol{W}_{\text {rotor }} \\
\text { After design space reduction }\end{array}$ \\
\hline \hline $\mathrm{R}^{2}$ & 0.987 & 0.917 & 0.996 & 0.995 \\
$\mathrm{R}^{2}$ adj & 0.985 & 0.905 & 0.996 & 0.994 \\
Root Mean Square Error & 0.094 & 0.020 & 0.0235 & 0.00170 \\
Mean of Response & 1.04 & 0.771 & 1.04 & 0.844 \\
Observations & 224 & 224 & 310 & 310 \\
\hline
\end{tabular}

\section{Acknowledgment}

This research is performed in coordination with the Institute for Future Space Transport (IFST) under the Constellation University Institute Project (CUIP). 


\section{References}

${ }^{1}$ Griffin, M. D., and French, J. R., Space Vehicle Design, American Institute of Aeronautics and Astronautics, Inc., Washington, DC, 1991, pp. 179-181.

${ }^{2}$ Karamanis, N., Martinez-Botas, R. F., "Mixed-flow turbines for automotive turbochargers: steady and unsteady performance," International Journal of Engine Research, Vol. 3, No. 3, Sept. 2002, pp. 127-138.

${ }^{3}$ Myers, R. H., and Montgomery, D. C., Response Surface Methodology, New York: John Wiley \& Sons, Inc., 2002.

${ }^{4}$ Papila, N., Shyy, W., Griffin, L., Huber, F. and Tran, K., "Preliminary Design Optimization for a Supersonic Turbine for Rocket Propulsion", AIAA/SAE/ASME/ASEE 35th Joint Propulsion Conference, CP-3242, July 16-19, 2000.

${ }^{5}$ Griffin, L. W. and Dorney, D. J., "RLV Turbine Performance Optimization," $12^{\text {th }}$ Annual Propulsion Engineering Research Center (PERC) Symposium, 2000.

${ }^{6}$ Papila, N., Shyy, W., Griffin, L., and Dorney, D.J., "Shape Optimization of Supersonic Turbines Using Global Approximation Methods," Journal of Propulsion and Power, Vol. 18, pp. 509-518, 2002.

${ }^{7}$ Madsen, J. I., Shyy, W., and Haftka, R. T., "Response Surface Techniques for Diffuser Shape Optimization," AIAA Journal, Vol. 38, pp. 1512-1518, 2000.

${ }^{8}$ Vaidyanathan, R., Papila, N., Shyy, W., Tucker, K. P., Griffin, L. W., Haftka, R. T., and Fitz-Coy, N., "Neural network and response surface methodology for rocket engine component optimization," 8th AIAA/USAF/NASA/ISSMO Symposium on Multidisciplinary Analysis and Optimization, CP-4480, 2000.

${ }^{9}$ Griffin, L., Dorney, D., Huber, F., Shyy, W., Papila, N. and Tran, K., "Detailed Aerodynamic Design Optimization of an RLV Turbine," 37th AIAA/ASME/SAE/ASEE Joint Propulsion Conference and Exhibit, CP-3397, 2001.

${ }^{10}$ Huber, F., "Turbine Aerodynamic Design Tool Development", Space Transportation Fluids Workshop, Marshall Space Flight Center, AL, April 2001.

${ }^{11}$ Sondak, D. L. and Dorney, D. J., "General Equation Set Solver for Compressible and Incompressible Turbomachinery Flows," AIAA 2003-4420, 39th AIAA/ASME/SAE/ASEE Joint Propulsion Conference and Exhibit, Huntsville, AL, July 20-23, 2003.

${ }^{12}$ Venkateswaran, S. and Merkle, C. L., "Analysis of Preconditioning Methods for the Euler and Navier-Stokes Equations, Von Karman Institute Lecture Series, March 8-12, 1999.

${ }^{13}$ Baldwin, B. S., and Lomax, H., "Thin Layer Approximation and Algebraic Model for Separated Turbulent Flow," AIAA Paper 78-257, Huntsville, AL, January, 1978.

${ }^{14}$ Oefelein, J. C., Sandia Corporation, Livermore, CA, Private Communication, December, 2002.

${ }^{15} \mathrm{http}: / /$ webbook.nist.gov/chemistry/fluid

${ }^{16}$ Dorney, D. J., Sondak, D. L. and Marcu, B., "Application of a Real-Fluid Turbomachinery Analysis to Rocket Turbopump Geometries," AIAA 2005-1007, 43rd AIAA Aerospace Sciences Meeting and Exhibit, Reno, NV, January 10-13, 2005.

17 McKay, M.D., R. J. Beckman, and W. J. Conover, "A Comparison of Three Methods for Selecting Values of Input Variables in the Analysis of Output from a Computer Code." Technometrics Vol. 21, No. 2, 1979, pp. 239245.

${ }^{18}$ Deb, K., Agrawal, S., Pratap, A., Meyarivan, T., "A fast and elitist multi-objective genetic algorithm for multiobjective optimization: NSGA-II.” Nature VI Conference, Paris, pp. 849-858, 2000.

${ }^{19}$ Goel, T., Vaidyanathan, R., Haftka, R. T., Queipo, N. V., Shyy, W., Tucker, P. K. "Response surface approximation of Pareto optimal front in multi-objective optimization" 10th AIAA/ISSMO Multidisciplinary Analysis and Optimization Conference, CP-4501, 2004.

${ }^{20}$ Knowles, J. D., Corne, D. W. "Approximating the Nondominated Front Using the Pareto Archived Evolution Strategy." Technical Report RUCS/1999/TR/005/A, June 1999.

${ }^{21}$ Sobol, I. M., "Sensitivity Analysis for Nonlinear Mathematical Models," Mathematical Modeling \& Computational Experiment, Vol. 1, No. 4, 1993, pp. 407-414.

${ }^{22}$ Homma, T. and Saltelli, A., "Importance Measures in Global Sensitivity Analysis of Nonlinear Models," Reliability Engineering and System Safety, Vol. 52, 1996, pp. 1-17.

${ }^{23}$ Saltelli A., Tarantola S., and Chan K., "A Quantitative Model-Independent Method for Global Sensitivity Analysis of Model Output," Technometrics, Vol. 41, No. 1, 1999, pp. 39-56. 